\title{
Time-Frequency Parameters of the Surface Myoelectric Signal for Assessing Muscle Fatigue during Cyclic Dynamic Contractions
}

\author{
Paolo Bonato*, Serge H. Roy, Marco Knaflitz, and Carlo J. De Luca
}

\begin{abstract}
The time-dependent shift in the spectral content of the surface myoelectric signal to lower frequencies has proven to be a useful tool for assessing localized muscle fatigue. Unfortunately, the technique has been restricted to constant-force, isometric contractions because of limitations in the processing methods used to obtain spectral estimates. A novel approach is proposed for calculating spectral parameters from the surface myoelectric signal during cyclic dynamic contractions. The procedure was developed using Cohen class time-frequency transforms to define the instantaneous median and mean frequency during cyclic dynamic contractions. Changes in muscle length, force, and electrode position contribute to the nonstationarity of the surface myoelectric signal. These factors, unrelated to localized fatigue, can be constrained and isolated for cyclic dynamic contractions, where they are assumed to be constant for identical phases of each cycle. Estimation errors for the instantaneous median and mean frequency are calculated from synthesized signals. It is shown that the instantaneous median frequency is affected by an error slightly lower than that related to the instantaneous mean frequency. In addition, we present a sample application to surface myoelectric signals recorded from the first dorsal interosseous muscle during repetitive abduction/adduction of the index finger against resistance. Results indicate that the variability of the instantaneous median frequency is related to the repeatability of the biomechanics of the exercise.
\end{abstract}

Index Terms-Cyclic contractions, electromyography (EMG), fatigue, muscle, spectral analysis, time-frequency analysis.

\section{INTRODUCTION}

$\mathbf{P}$ IPER showed at the beginning of this century [32] that during a sustained muscle contraction the spectral components of the surface myoelectric signal are compressed toward the lower frequencies. The mechanisms that regulate this phenomenon have only been clarified during the last two decades [3]. Following these developments, researchers have pursued the analysis of the surface myoelectric signal for such clinical problems as muscular dystrophy [20], [33] and lower back pain [4], [25], [36] as well as for gaining knowledge about the physi-

Manuscript received January 7, 1998; revised April 6, 2001. This work was supported in part by The Whitaker Foundation under Project "Assessment of muscle function during cyclical lifting through the analysis of surface electromyographic signals." Asterisk indicates corresponding author.

*P. Bonato is with the NeuroMuscular Research Center, Boston University, 19 Deerfield Street, Boston, MA 02215 USA (e-mail: pbonato@bu.edu).

S. H. Roy and C. J. De Luca are with the NeuroMuscular Research Center, Boston University, Boston, MA 02215 USA.

M. Knaflitz is with the Dipartimento di Elettronica, Politecnico di Torino, 10100 Torino, Italy.

Publisher Item Identifier S 0018-9294(01)05132-1. ological mechanisms underlying fatigue and motor unit control [19], [24].

Previous studies of muscle fatigue based on the surface myoelectric signal have been limited to data collected during constant-force contractions sustained isometrically [11]. In this contraction paradigm, the main phenomenon that influences the frequency scaling of the power spectrum of the myoelectric signal is the accumulation of biochemical byproducts within the muscle [3]. The metabolite accumulation induces a progressive modification of the interstitial fluid $\mathrm{pH}$, which in turn causes a reduction of the propagation velocity of the action potential along the muscle fibers. This complex of electrophysiological phenomena is referred to as localized muscle fatigue [11].

When the surface myoelectric signal is recorded during a constant-force, isometric contraction, it may be considered as a realization of a wide-sense stationary stochastic process with Gaussian amplitude distribution and zero mean [3]. Under these conditions the correlogram or the periodogram may be adopted to estimate the power spectrum of the signal [30]. Either the median frequency of the power spectrum of the signal or its mean frequency is typically used to track the spectral scaling resulting from the progression of fatigue [11].

Although the analysis of the surface myoelectric signal detected during constant-force isometric contractions has been useful in a number of applications, tests which better represent "real-life" conditions are necessary for extending its applicability in the clinical and ergonomic sciences [6]. There is a need to study contractions that depart from constant-force isometric conditions, i.e., those in which the muscle force and the position of body segments change during the exercise. Throughout this paper, this contraction modality is referred to as dynamic.

When the surface myoelectric signal is recorded under dynamic conditions, the assumption of stationarity does not hold because the frequency content of the signal continuously changes over time. Nonstationarities of the surface myoelectric signal can be classified as slow or fast. Slow nonstationarities are mostly due to the accumulation of metabolites that causes the electrical manifestations of muscle fatigue. Fast nonstationarities are mainly related to the biomechanics of the task. Variations in muscle force cause a modification of the frequency content of the signal [5], [7]. A scaling of the power spectrum is also observed as a function of the change in muscle length [16]. In addition, the movement of the body segments causes a relative displacement of the electrodes with respect to the underlying muscle fibers, which affects the frequency content 
of the myoelectric signal [12], [22]. Therefore, the study of the frequency content of the myoelectric signal under dynamic conditions must be performed by methods that are appropriate for processing nonstationary signals. Cohen class time-frequency transforms have recently been shown suitable for processing surface myoelectric data recorded during dynamic contractions [6], [18], [21], [37]. Despite this advancement, further work is needed to define and validate parameters that can be used to monitor changes in the frequency content of the myoelectric signal during the cyclic exercise.

The aim of this paper is to describe recent advances on the use of time-frequency transforms for assessing localized muscle fatigue during cyclic dynamic contractions. First, we describe recommendations for calculating the instantaneous median and mean frequency parameters, based on previous work using time-frequency transforms of the Cohen class. Then, we demonstrate the estimation error that affects these instantaneous frequency parameters. The emphasis is placed on contractions that are cyclically generated. We propose and discuss methods to increase the stability of the instantaneous frequency parameter estimation in order to make the assessment of localized fatigue more reliable. A sample application to the analysis of real surface myoelectric signals is presented for the first dorsal interosseous muscle during repetitive abduction/adduction of the index finger against resistance. The progressive scaling of the time-frequency spectrum of the myoelectric signal toward lower frequencies is described and then discussed in terms of its applicability to measuring localized muscle fatigue.

\section{Cohen-Class Time-Frequency ANalysis of NONSTATIONARY PROCESSES}

Among the different possible approaches to the analysis of nonstationary signals, Cohen class transformations [9] have received considerable attention, particularly in biomedical signal processing [1]. This class of time-frequency representations is particularly suitable to analyze surface myoelectric signals recorded during dynamic contractions, which may be modeled as realizations of nonstationary stochastic processes [6], [18], [21], [37]. Previous work by Martin and Flandrin [26], [27], Amin [2], and Sayeed and Jones [37] demonstrated that any Cohen class time-frequency spectrum $S(t, f)$ may be written as

$$
\begin{aligned}
S(t, f)= & \int_{-\infty}^{\infty} \int_{-\infty}^{\infty} \int_{-\infty}^{\infty} E\left\{x\left(t^{\prime}+\tau / 2\right) x^{*}\left(t^{\prime}-\tau / 2\right)\right\} g(\theta, \tau) \\
& \cdot e^{-j 2 \pi \theta\left(t^{\prime}-t\right)} e^{-j 2 \pi f \tau} d \theta d t^{\prime} d \tau
\end{aligned}
$$

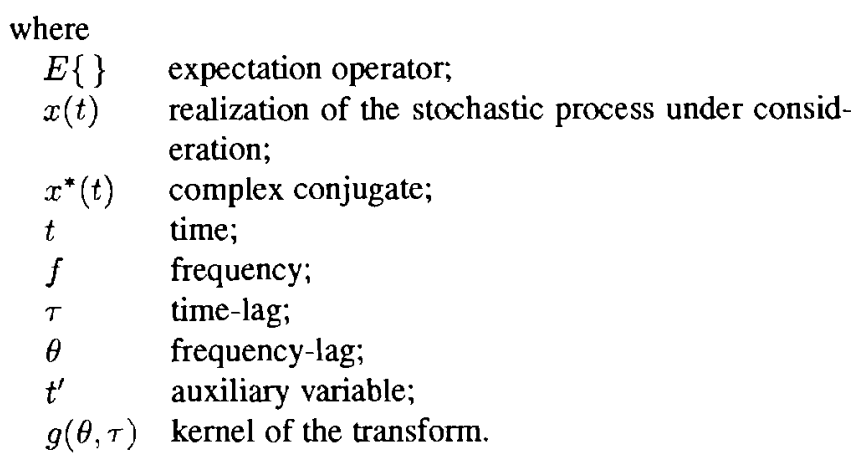

Also, $x(t)$ indicates the analytic process obtained from the real recording. Since the kernel $g(\theta, \tau)$ does not depend on $t$ and $f$, the resulting distribution is time and frequency shift invariant [9]. This characteristic is of paramount importance when correlating the time-frequency representation with physical or physiological phenomena and makes Cohen class distributions particularly suitable to study muscle fatigue.

When one chooses $g(\theta, \tau)=1$, the resulting distribution is referred to as Wigner-Ville distribution [9]. The Wigner-Ville is optimal to analyze signals constituted by a single component. However, it is not well suited for application to multicomponent signals, since the bilinearity of the transform induces the presence of interference terms [9]. Several authors attempted to reduce the interference terms by developing specific kernels. In the following, we will investigate the application of the Choi-Williams and the Born-Jordan transforms. The Choi-Williams transform [8] has been shown to be particularly suitable for the analysis of surface myoelectric signals [6], [18], [21], [37]. Its kernel is defined as $g(\theta, \tau)=e^{-(2 \pi \theta \tau)^{2} / \sigma}$. In the analysis of surface myoelectric signals recorded during dynamic contractions the parameter $\sigma$ has been chosen equal to 1 as this value appeared to be satisfactory for processing myoelectric data [6], [18], [21], [37]. This is also the way the kernel was used in this study. The Born-Jordan transform provides an estimate of the time-frequency spectrum that minimizes the average variance for white noise processes [14]. Its kernel is defined as $g(\theta, \tau)=\sin (\pi \theta \tau) /(\pi \theta \tau)$.

Sayeed and Jones [37] demonstrated that the formulation presented in (1) may also be utilized when a single realization of the analyzed stochastic process is available, as is the case when processing surface myoelectric signals recorded during dynamic contractions. Therefore the expectation operator may be removed. However, when removed, the variance of the timefrequency spectrum estimate may be too high to derive parameters measuring localized muscle fatigue. Averaging the distribution over finite time intervals [28], [40] decreases the estimation variance and does not introduce a significant bias if the averaging is limited to quasi-stationarity intervals [26], [27]. If the quasi-stationarity condition cannot be satisfied, setting the length of the time intervals for the averaging technique leads to a tradeoff between bias and variance of the estimate. When the averaging technique is applied, $D S(i, j)[D S(i, j) 1<i<N$ and $1<j<M$ being the time-frequency spectrum obtained by considering the discrete counterpart of (1)] is replaced in the time intervals $((k-1) P+1, k P), 1 \leq k \leq N / P$ by

$$
D S(k, j)=\frac{1}{P} \sum_{l=1}^{P} D S(l+(k-1) P, j) .
$$

This formula can be modified when overlapping among the finite time intervals is allowed. This approach is advantageous when one wants to avoid excessively decreasing the time resolution of the averaged distribution and still apply the averaging over a large enough window to decrease the estimation variance.

Once the time-frequency spectrum of the myoelectric signal is obtained, the instantaneous median and mean frequency may be defined by replacing the power density spectrum with the time-frequency spectrum in the formulae that define the corresponding stationary parameters [11]. Since the instantaneous 
median and mean frequency are extensions of the parameters defined in the stationary case, their physiological interpretation is parallel with that of the corresponding parameters defined in the stationary case.

Several factors may cause a sudden change in the frequency content of the surface myoelectric signal when the biomechanics of the task may vary suddenly. Thus, the estimation error has to be insensitive to changes in the instantaneous frequency spectrum. In addition, the estimator must be insensitive to instantaneous variations of the signal-to-noise ratio (SNR). In fact, when the force exerted by the muscle under investigation changes, the signal to noise ratio varies accordingly. It follows that desirable estimators should track actual variations of the observed parameters minimizing the influence of variations of the signal to noise ratio.

A way to minimize the sensitivity of the instantaneous frequency parameter estimation to additive noise is to avoid the integration of the high frequency portion of the time-frequency representation where there are no significant signal components. In order to implement this approach, we adopted an algorithm previously suggested by D'Alessio [10] to estimate the upper frequency of the power density spectrum. We used then the upper frequency to limit the considered frequency range when computing the instantaneous frequency parameters.

The estimation procedure may be therefore summarized as follows.

I. Estimate the time-frequency spectrum using the discrete time counterpart of (1).

II. Average over short time intervals of $P$ samples, according to (2).

III. Compute the upper frequency according to D'Alessio's algorithm [10].

IV. Estimate the instantaneous mean and median frequency defined as follows:

$$
\begin{aligned}
\sum_{j=1}^{I M D F(k)} D S(k, j) & =\sum_{j=I M D F(k)}^{U F(k)} D S(k, j) \\
I M N F(k) & =\frac{\sum_{j=1}^{U F(k)} k \delta f D S(k, j)}{\sum_{j=1}^{U F(k)} D S(k, j)}
\end{aligned}
$$

where

$I M D F(k)$ and $I M N F(k)$ instantaneous median and mean frequency;

$U F(k) \quad$ denotes the upper frequency;

$D S(k, j) \quad$ time averaged estimate of the

time-frequency spectrum;

$\delta f \quad$ frequency interval between the samples of the time-frequency spectrum;

$k$ and $j \quad$ time and frequency indexes, respectively.

\section{Characterization OF THE Estimation ERROR}

Computer synthesized signals were used to assess the application of the above-described procedure to the surface myo-
A

B
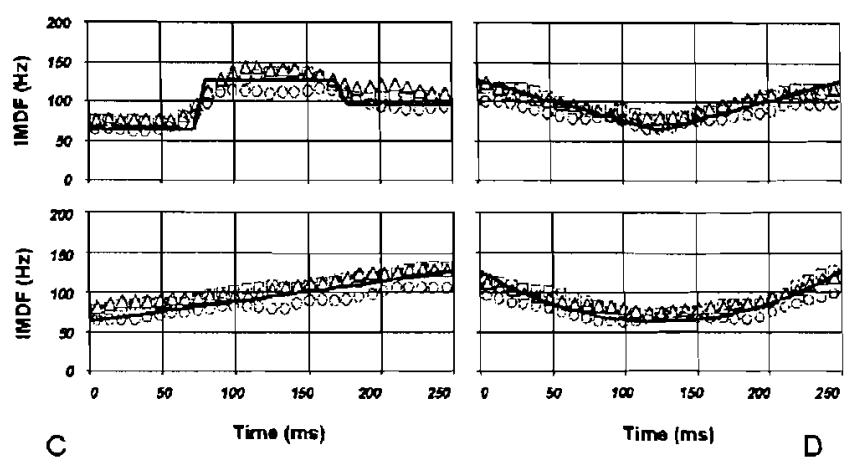

Fig. 1. Instantaneous mean frequency of different nonstationary stochastic processes: expected value (continuous lines) and estimates obtained by different time-frequency transforms (circles are relative to the Wigner-Ville transform, triangles to the Bom-Jordan transform, and squares correspond to the Choi-Williams transform). The simulated nonstationarities are (a) step, (b) triangular, (c) linear increasing, and (d) quadratic profile.

electric signal recorded during dynamic contractions. The synthesized signals were obtained by bandpass filtering a white Gaussian series. The bandpass filter was chosen according to previous work by Stulen and De Luca [39] who suggested simulating surface myoelectric signals using a shaping filter with the following transfer function:

$$
H(f)=\frac{k^{2} f_{h}^{2} f^{2}}{\left(f^{2}+f_{l}^{2}\right)\left(f^{2}+f_{h}^{2}\right)^{2}}
$$

where

$f_{l} \quad$ bandpass low cutoff frequency;

$f_{h} \quad$ bandpass high cutoff frequency;

$k \quad 1.699 / f_{h}$.

Following Stulen and De Luca's recommendations, the high cutoff frequency was chosen equal to twice the low cutoff frequency.

To apply the shaping filter to the white Gaussian series, the transfer function was transformed in the discrete domain by means of a bilinear transformation and the corresponding infinite impulse response filter was derived. Variations to the frequency content of the simulated surface myoelectric signal were produced by incrementally adapting the filter's coefficients every 16 samples. A sampling rate equal to 1024 $\mathrm{Hz}$ was simulated.

We considered four different profiles of the instantaneous median and mean frequency in which fast variations of the spectral characteristics were simulated. The four theoretical profiles are shown as solid lines in Fig. 1. In all these cases, dramatic changes in the frequency content of the signal were simulated within time intervals as short as $250 \mathrm{~ms}$. Two additional stationary intervals of $125 \mathrm{~ms}$ each (not shown in Fig. 1) were generated before and after the nonstationary interval. The statistical properties of the initial and final stationary sequences were kept equal to those of the initial and terminal part of the nonstationary series. Epochs lasting $0.5 \mathrm{~s}$ were generated in this manner, resulting in time-frequency distributions with a frequency resolution equal to $2 \mathrm{~Hz}$. The time-frequency transformation was implemented by first deriving the instantaneous autocorrelation function using an alias-free algorithm and then applying the 
kernel of the transformation in the ambiguity domain [6]. When computing the instantaneous autocorrelation function, the timesupport was kept equal to $0.5 \mathrm{~s}$ and the time-lag support was limited to $250 \mathrm{~ms}$. The Fourier transform (from $t$ to $\theta$ ) was taken and the kernel of the transform was applied. The time-frequency representation was computed by an inverse Fourier transform (from $\theta$ to $t$ ) and then Fourier transforming (from $\tau$ to $f$ ) the function derived in the $(t, \theta)$ domain. Zero-padding from 512 to 1024 samples was applied before Fourier transforming (from $\tau$ to $f$ ). This procedure allowed us to estimate the time-frequency distribution with frequency resolution of $2 \mathrm{~Hz}$ and frequency interval of $1 \mathrm{~Hz}$. In the time-frequency domain, we did not consider the Fourier transform corresponding to the first and last 128 samples on the time axis because the estimation errors in such intervals increase due to the finite time support of the input signal. The rest of the distribution was averaged over finite time intervals of 32 samples using a version of (2) modified in order to allow $75 \%$ overlap among time intervals. Ultimately, (3) and (4) were utilized to compute the instantaneous median and mean frequency.

For each profile type, one-hundred realizations of the nonstationary stochastic process were considered. Three time-frequency transformations were applied to each epoch, i.e., the Wigner-Ville, the Choi-Williams, and the Born-Jordan transformations [1].

Fig. 1 shows the simulated profiles of the instantaneous mean frequency (solid lines), which are compared to the estimates derived by applying the three different time-frequency distributions mentioned above. Fig. 1(a) is an example of abrupt changes in the frequency content of the myoelectric signal. It is apparent that the bias of the estimate of the instantaneous mean frequency for the Choi-Williams distribution is always smaller than the bias for the Wigner-Ville and the Born-Jordan transforms. This is most likely due to the superior capability of the Choi-Williams distribution in rejecting the interference terms. Fig. 1(b)-(d) shows that the bias depends on the specific simulated profile, although the lowest and highest expected frequencies are equal in every profile. This dependency is likely due to the presence of interference terms, whose geometry depends on the specific profile. Furthermore, Fig. 1 shows that the time-averaging window utilized to decrease the variability of the estimate of the time-frequency spectrum, as well as the kernel of the Bom-Jordan and Choi-Williams transforms, produce a slight smoothing of the time course of the estimated mean frequency.

In Fig. 2, the simulated profiles of the instantaneous median frequency (solid lines) are compared to the estimates derived from the three different time-frequency distributions. The behavior of the instantaneous median frequency is similar to that of the mean frequency. From the results it may be argued that the instantaneous median frequency obtained by using the Choi-Williams transform is associated with a smaller bias on average than the estimates derived by means of the Bom-Jordan and the Wigner-Ville distributions.

To evaluate the effect of different SNRs, the analysis was repeated by superimposing an uncorrelated zero-mean white Gaussian process on the signal, which resulted in SNRs equal to $10 \mathrm{~dB}$ and $1 \mathrm{~dB}$. A comparison was made among profiles re-
A B
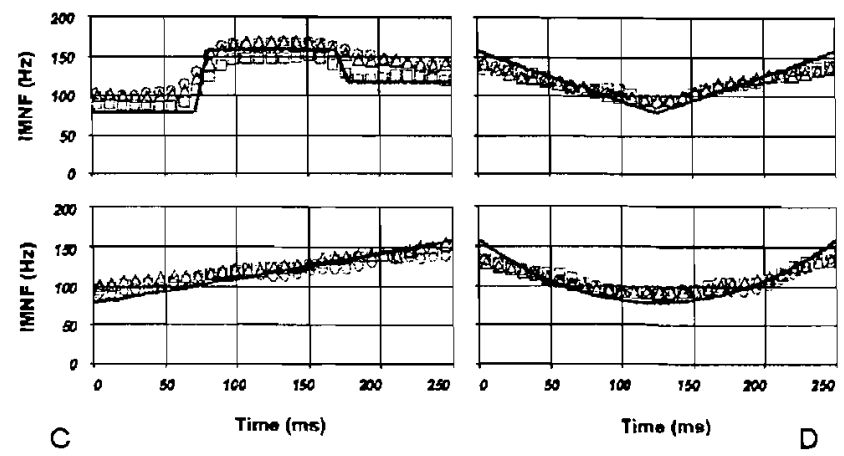

Fig. 2. Instantaneous median frequency of different nonstationary stochastic processes: expected value (continuous lines) and estimates obtained by different time-frequency transforms (circles are relative to the Wigner-Ville transform, triangles to the Born-Jordan transform, and squares are relative to the Choi-Williams transform). The simulated nonstationarities are (a) step, (b) triangular. (c) linear increasing, and (d) quadratic profile.
A

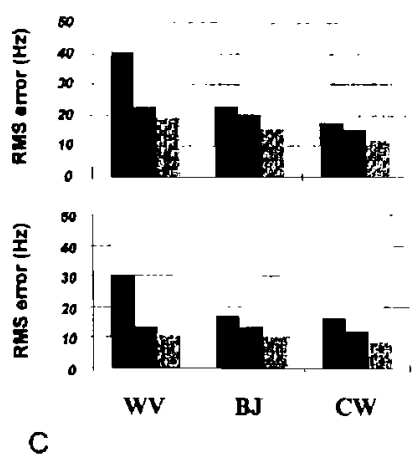

B
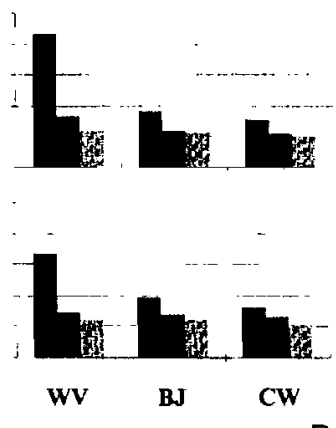

Fig. 3. Root-mean-square error of the reconstructed profiles of the instantaneous mean frequency. Data relative to the instantaneous mean frequency obtained by utilizing the Wigner-Ville (WV), the Born-Jordan (BJ), and the Choi-Williams (CW) transform are reported. For each transform three bars are represented, relative to a SNR equal to $1 \mathrm{~dB}$ (bar on the left side), $10 \mathrm{~dB}$ (bar in the middle), and infinite, i.e., no-noise (bar on the right side), respectively. Different panels correspond to different profiles according to those reported in Fig. 1.

constructed by means of different time-frequency transformations by computing the root-mean-square error (rmse) associated with each profile and SNR. Figs. 3 and 4 present the results. For each plot, the three different bars indicate the results for SNRs equal to $1 \mathrm{~dB}, 10 \mathrm{~dB}$, and infinity (no noise), respectively. The results show the higher error resulting from the application of the Wigner-Ville transform, particularly when the SNR is equal to $1 \mathrm{~dB}$. On average, the mse associated with the Wigner-Ville transform was found to be $50 \%$ greater than that associated with the Born-Jordan transformation and $75 \%$ greater than that associated with the Choi-Williams transformation. Moreover, a comparison between Figs. 3 and 4 shows that the rmse that marks the reconstructed profile of the instantaneous median frequency is lower than that of the instantaneous mean frequency. On average the reconstructed profile of the instantaneous median frequency was associated with a rmse $18 \%$ lower than the corresponding profile of the instantaneous mean frequency. Figs. 3 and 4 also indicate that the sensitivity 


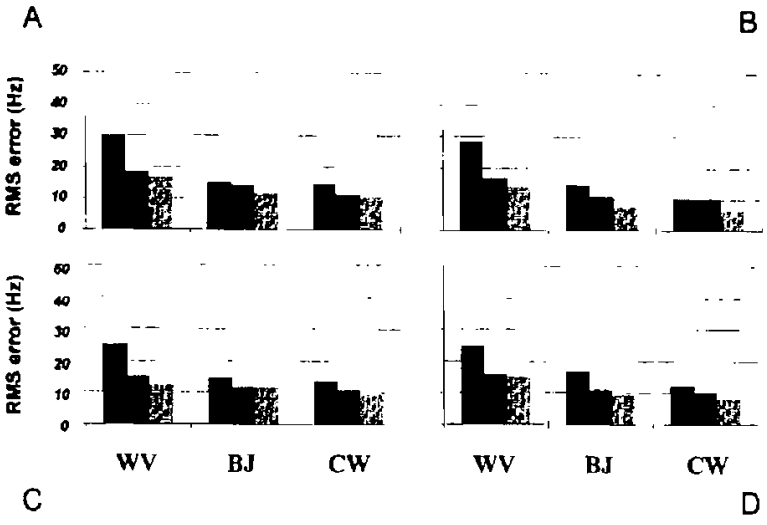

Fig. 4. Root-mean-square error of the reconstructed profiles of the instantaneous median frequency. Data relative to the instantaneous median frequency obtained by utilizing the Wigner-Ville (WV), the Bom-Jordan (BJ), and the Choi-Williams (CW) transform are reported. For each transform three bars are represented relative to a SNR equal to $1 \mathrm{~dB}$ (bar on the left side), $10 \mathrm{~dB}$ (bar in the middle), and infinite, i.e., no-noise (bar on the right side), respectively. Different panels correspond to different profiles according to those reported in Fig. 2.

to additive noise of the instantaneous median frequency is lower than that of the instantaneous mean frequency, particularly when the Wigner-Ville transform is considered. From these comparisons we concluded that the Choi-Williams distribution produces reconstructed profiles with the lowest error, although the Born-Jordan distribution also gives acceptable results. As suggested in previous works [6], [18], [21], [37], this finding can be related to the high selectivity of the kernel of the Choi-Williams transform that results in a more efficient attenuation of the interference terms.

Results from the simulated myoelectric signals suggest that the Choi-Williams transform is preferable to the Born-Jordan and the Wigner-Ville transforms when estimating the instantaneous median and mean frequency. Moreover, the reconstructed profile of the instantaneous median frequency is affected by a rmse lower than that related to the instantaneous mean frequency. Therefore, in the remainder of the work presented here, only the instantaneous median frequency estimates derived by the Choi-Williams transformation of the surface myoelectric signal will be considered. Bias and standard deviation were computed for this specific case and all the profiles and SNRs. For the Choi-Williams transform, biases ranged between $1.7 \mathrm{~Hz}$ and $10.7 \mathrm{~Hz}$ and standard deviation values ranged between $21.6 \mathrm{~Hz}$ and $32.4 \mathrm{~Hz}$. The relatively high values of the standard deviation of the estimates demonstrate that further processing methods must be developed to obtain estimation errors acceptable for research and clinical applications. This problem is addressed in Section IV.

\section{IMPROVING ESTIMATION STABILITY: THE IMPORTANCE OF CyClic CONTRACTIONS}

Strategies are needed to improve the sensitivity of time-frequency estimates to the myoelectric spectral shift resulting from localized muscle fatigue during dynamic contractions. One method adopted was to limit the analysis to a dynamic activity that is mechanically reproducible. Therefore, only repetitive cyclic contractions that result in periodic mechanical changes to the muscle were considered. It is assumed that the cyclic task results in nearly identical changes in muscle force, muscle length, and velocity of shortening/lengthening of the muscle fibers.

Cyclic contractions may be seen as subdivided into two classes: 1) highly fatiguing contractions, such as those that require a maximal or near maximal effort and can only be repeated for relatively few cycles; or 2) slowly fatiguing contractions that are performed at a relatively low percentage of maximal effort but are sustained at a relatively high repetition rate. This paper focuses on slowly fatiguing contractions because they are likely to be more common in daily activities. To improve the stability of the time-frequency parameter estimation for such contractions, we propose a procedure based on the following points: 1) only a limited part of the myoelectric signal "burst" corresponding to a fixed portion of the contraction cycle is analyzed and 2) the instantaneous time-frequency parameters can be averaged for a few consecutive cycles because the myoelectric signal is considered to be quasi-cyclostationary. The first point is proposed in order to reduce the effect of biomechanical factors unrelated to fatigue that can modify the time-frequency estimates. The second point leads to a significant decrease of the standard deviation of the estimates, which affects the time-frequency parameter estimation.

The proposed approach to analyze cyclic dynamic contractions consists of taking identical portions of the myoelectric signal "burst" during which the effects of muscle force, muscle length, and movement of the electrodes with respect to the active muscle fibers are assumed be the same across cycles. If we assume that these factors are invariant for the same portion of each cycle, then the changes in the time-frequency spectra of the surface myoelectric signal monitored from these portions should be closely related to the spectral compression resulting from fatigue processes. If no other strategy is adopted, however, the instantaneous median frequency estimates will likely be affected by a high standard deviation, as observed from the simulations illustrated in Section III. Therefore, assuming that changes in the frequency content of the myoelectric signal are negligible within a few cycles, averaging across cycles can reduce the variability of the instantaneous median frequency. When doing so, it is assumed that the surface myoelectric signal may be modeled as a cyclostationary process within these cycles, i.e., a stochastic process whose first and second moments are marked by a periodic behavior over an indefinite period of time [13]. This assumption is valid in our application within a limited time interval (i.e., within a limited number of cycles). Under these conditions the surface myoelectric signal is referred to as quasi-cyclostationary.

\section{Sample Application to Real Data}

To demonstrate the effectiveness of the method proposed in this paper, a sample application is presented for surface myoelectric signals recorded from the first dorsal interosseous muscle during a cyclic exercise. The task, repeated abduction/adduction of the index finger, was selected because of its biomechanical simplicity and ease of constraining the movement. The first dorsal interosseous muscle is the only 


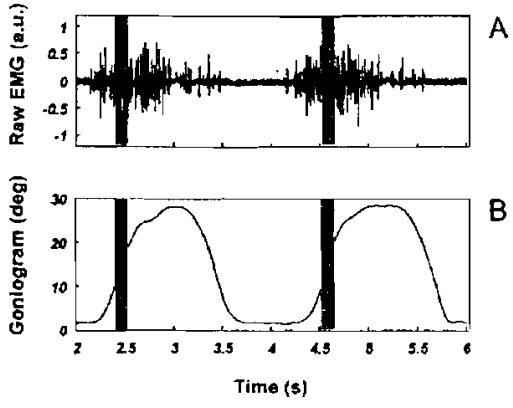

Fig. 5. Schematic representation of the selection procedure for windowing the surface myoelectric signal. (a) Raw surface myoelectric signal recorded during two successive repetitions of abduction/adduction of the index finger. (b) Joint angle that represents the index finger position during the exercise. An output corresponding to $0^{\circ}$ signifies the index finger in the neutral position (close to the middle finger).

prime mover active during the abduction/adduction of the index finger [3]. These factors can minimize unpredictable variations in the time-course of the estimated frequency parameter related to the variability of the biomechanics.

A custom apparatus was built to provide abduction/adduction of the index finger under constant-load conditions throughout the entire range of the exercise. The device consists of a plate to constrain the thumb and middle finger in a fixed position and maintain the wrist in neutral. The index finger is free to move in a range from $0^{\circ}$ (neutral position) to $30^{\circ}$ of abduction. A constant adduction load is applied to the middle phalanx of the index finger during the exercise by a weight attached to a pulley system. The load was kept constant at a level equal to ten percent of maximum voluntary contraction performed at $0^{\circ}$. A potentiometer was used to measure the metacarpal joint angle during abduction/adduction of the index finger. A visual display was added to allow the subject to pace the exercise and limit each cycle to a duration of $2 \mathrm{~s}$. The exercise was performed for $2.5 \mathrm{~min}$, which resulted in seventy-five repetitions of the abduction/adduction movement. This protocol provided a moderately fatiguing task to the subject.

The surface myoelectric signal was detected continuously during the exercise by an array of electrodes consisting of seven parallel silver bars $[0.5 \mathrm{~mm}$ wide; $0.5 \mathrm{~cm}$ long] with an inter-electrode separation of $5 \mathrm{~mm}$. Single differential signals were recorded from adjacent parallel bars and amplified at a gain of 1000 and band-passed at $8-450 \mathrm{~Hz}$. The signals were sampled at a rate of $1024 \mathrm{~Hz}$ using a PC workstation with a 12-bit analog-to-digital signal processing card (Microstar DAP-3200). The position of the innervation point was detected graphically as illustrated in previous work [30]. The single differential signal derived from the pair of bars corresponding to the mid-point between the innervation zone and the tendon on the distal portion of the first dorsal interosseous muscle was selected for further analysis according to a previously published method [34].

Fig. 5 presents the technique that was utilized to segment the myoelectric bursts. Fig. 5(a) depicts the surface myoelectric signal recorded during cyclic abduction/adduction of the index finger while constrained to a single plane using the custom mechanical device. Fig. 5(b) indicates the angle formed by the
A
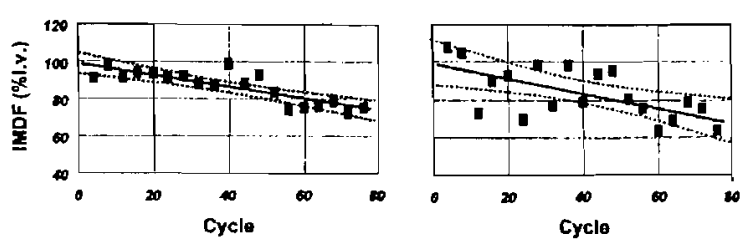

Fig. 6. Time course of the instantaneous median frequency during repeated abduction/adduction of the index finger. Markers are relative to the estimates performed by selecting (a) the most and (b) the least biomechanically repeatable portion of each repetition of the exercise (see text for details). For each plot, the continuous line was obtained by linear interpolation of the measurements reported by the markers. The dotted lines indicate the intervals at $95 \%$ confidence level. Data were nomalized according to the intercept with the $y$ axis of the regression line (i.y.-initial value) to obtain the percentage decrease in the time-frequency parameter during the exercise.

index finger with respect to the neutral position. The shadowed vertical bands identify the portion of the burst considered for analysis in each cycle. The initial point of this time interval was set by a threshold equal to $10^{\circ}$ of the joint angle. A window of 512 samples was applied to the surface myoelectric signal and the time-frequency spectrum was estimated. A time-averaging window was utilized to decrease the variability of the estimate of the time-frequency spectrum. A single value of the estimate corresponding to the mid-point of the applied window was calculated for deriving the instantaneous median and mean frequency.

The threshold value of $10^{\circ}$ was selected because it was the most repeatable portion of the joint angle trajectory. Repeatability was assessed by considering eleven threshold values be tween $2.5^{\circ}$ and $27.5^{\circ}\left(2.5^{\circ}\right.$ apart). The average trajectory during short time intervals selected from each threshold value was computed. The time interval corresponded to the length of the timeaveraging window applied to the time-frequency distribution. The rmse was estimated for each time interval by considering the average trajectory as the true value and evaluating the error over the entire duration of the cyclic exercise.

The threshold value with the least error was utilized to select the surface myoelectric signal segment to be analyzed by means of the Choi-Williams transform. The time-course of the instantaneous median frequency was used to monitor the spectral compression to lower frequencies during the exercise. Fig. 6(a) depicts this time-course for portions of the cycle selected for processing. A progressive decrease of the instantaneous median frequency during the entire exercise is evident and indicative of myoelectric fatigue.

Each estimate was obtained by computing the time-frequency spectrum utilizing the Choi-Williams transform and applying a time-averaging window of 32 samples. Quasi-cyclostationarity was assumed over four consecutive repetitions (i.e., cycles) of the exercise. Therefore each sample reported in Fig. 6(a) was obtained as the average of four estimates of the instantaneous median frequency. Averaging the values obtained from four consecutive cycles of the task reduced the standard deviation of the instantaneous median frequency to almost half the value obtained before averaging.

Fig. 6(b) demonstrates the increased variability of the instantaneous median frequency when the biomechanics minimalvariability criterion is not used to select the portion of the cycle 
considered for analysis. In this example, the threshold value was set to $25^{\circ}$. It is worthwhile noting that the rmse of the joint angle trajectory for this threshold value was approximately twice that corresponding to a threshold value of $10^{\circ}$. This result emphasizes the importance of considering the time interval in which the variability of the biomechanics of the exercise is the smallest.

\section{DISCUSSION}

This paper describes recent developinents in advancing a novel procedure to track the spectral scaling of the myoelectric signal during repetitive cyclic contractions. The proposed approach was developed to address the drawbacks of traditional approaches that segment the myoelectric signal into short epochs. The use of short windows to segment the myoelectric signal yields a poor frequency resolution of the spectral estimates [30]. Theoretical work by Hof [15] has shown that the estimation error of the median and mean frequency is inversely proportional to the length of the window utilized to segment the myoelectric signal. Work by Merletti et al. [29] using computer simulations has supported these findings. It is well recognized [6], [15], [29] that the use of short segments yields an error too large to assess changes in the frequency content of the surface myoelectric signal associated with localized fatigue during dynamic contractions. An alternative approach that addresses the frequency resolution limitation inherent in selecting a short time window is to compute the square magnitude of the Fourier transform of each burst of the surface myoelectric signal, disregarding the nonstationarities evolving within the burst itself. This technique estimates the frequency marginal of the time-frequency representation. Therefore, when computing the mean frequency of the square magnitude of the Fourier transform of the signal, an estimate of the frequency coordinate of the centroid of the time-frequency distribution is obtained. This value roughly corresponds to the average of the instantaneous mean frequency estimates. Similarly, the estimate of the median frequency performed on the square magnitude of the Fourier transform of the signal roughly corresponds to the average of the instantaneous median frequency estimates. If localized muscle fatigue leads to a simple scaling of the entire time-frequency representation of the surface myoelectric signal, the results obtained by estimating the frequency parameters through the Fourier transform of each burst of the signal approximately correspond to the changes in instantaneous median and mean frequency observed by the time-frequency analysis. We did not choose to adopt this alternative procedure because it assumes that the factors unrelated to fatigue that affect the surface myoelectric signal during the cyclic activity (e.g., changes in muscle length, muscle force, and electrode position) are perfectly repeatable during each cycle of the exercise. This assumption is unlikely because of the difficulty in controlling all the biomechanical variables of the exercise while muscular fatigue progresses. It would be necessary to completely constrain the biomechanical conditions of the exercise to achieve such levels of repeatability.

The time-frequency transform that we adopted enabled us to select and analyze a specific portion of the exercise cycle that was biomechanically repeatable. In order to establish a relationship between the time-frequency characteristics of the surface myoelectric signal and the biomechanical changes occurring during the dynamic exercise, it was necessary to monitor the kinematics of the task. There is considerable evidence in the literature that length [16], force [5], [7], and electrode position [23] have a primary influence on the surface myoelectric signal during dynamic conditions. It was beyond the scope of this paper to selectively describe the respective influences of each of these factors. Instead, by selecting a repetitive, cyclical task, we pre-supposed that these factors were constrained to be the same for subsequent repetitions of the exercise. We also suggested that surface myoelectric signals recorded during dynamic cyclical contractions are quasi-cyclostationary within a few cycles. Unfortunately to our knowledge there is no method to measure the quasi-cyclostationarity of the surface myoelectric signal. A possible approach could be based on the computation of the distance [22] among portions of the time-frequency distributions of the myoelectric data recorded during consecutive cycles. Further studies are needed to investigate possible approaches to measure the quasi-cyclostationarity of the surface myoelectric signal and verify this assumption under complex conditions.

The method we adopted is somewhat akin to using isometric test contractions to briefly interrupt a dynamic activity and sample the myoelectric fatigue state of the muscle. Under such conditions, the influence of muscle force, velocity, and length are assumed to be constant [12]. The method of using test contractions is only a partial solution because it can introduce additional fatigue to the task being studied, it may be impractical to perform during real-life activities, and more importantly, it may result in sampling different populations of motor units than those being recruited during the dynamic activity. No studies to date have determined how serious these drawbacks are. The time-frequency approach described in this paper may provide the signal processing tools needed in future work to make such a comparison.

The sample application of the technique herein proposed for the analysis of surface myoelectric signals recorded from the first dorsal interosseous muscle demonstrates that this procedure may be useful for quantifying the spectral scaling that occurs as a result of fatigue during cyclic dynamic activities. The exercise task studied in this paper was carefully selected to improve the likelihood of success. The contractions involved a single prime mover, the range of muscle length change was relatively small, the position of the hand was well constrained, and the contractions followed a fixed and repetitive trajectory in which changes in electrode position, force, and length were likely repeatable. Further study will be necessary to correlate these spectral changes to biochemical and contractile indexes of muscle fatigue and to demonstrate more explicitly that the spectral scaling is a valid estimator of underlying physiological and mechanical measures of fatigue during dynamic contractions. Preliminary studies in which 31P-NMR spectroscopy was combined with surface myoelectric recordings during a fatiguing cyclical contraction have provided encouraging results [35]. Further development of the technique described in this paper will be needed to assess more complex activities which 
are not as constrained. This will be particularly desirable for many possible applications in the fields of ergonomics, rehabilitation, and sports medicine.

\section{CONCLUSION}

This paper provides the first comparison between instantaneous mean and median frequency parameters for assessing localized muscle fatigue during dynamic contractions. By using synthesized surface myoelectric signals, we demonstrated that the instantaneous median frequency is preferable to the instantaneous mean frequency because of its lower estimation error. We also demonstrated the benefit of the time-frequency transform of the Cohen class (i.e., the Choi-Williams transform) by comparing the estimation errors associated with the Wigner-Ville, the Bom-Jordan, and the Choi-Williams transforms. In order to obtain a satisfactory sensitivity of the instantaneous median frequency to changes in the surface myoelectric signal related to localized fatigue during dynamic cyclic contractions, we proposed two strategies: 1) limit the analysis of the surface myoelectric signal to the most biomechanically repeatable portion of the cycle and 2) average the instantaneous median frequency values across a few cycles where the assumption of quasi-cyclostationarity of the surface myoelectric signal is valid. Finally, we demonstrated a relationship between variability in the biomechanics of the task and variability of the instantaneous median frequency time-course during the exercise. This relationship emphasizes the importance of choosing the most repeatable portion of the cyclic task for assessing localized muscle fatigue.

We believe that the material presented in this paper may help extend the applicability of current myoelectric spectral analysis procedures to new areas. The possibility of monitoring the electrical manifestations of muscle fatigue noninvasively during dynamic activities will have important applications in the fields of rehabilitation, ergonomics, and sports medicine.

\section{ACKNOWLEDGMENT}

The authors would like to thank E. Kupa and L. D. Gilmore whose contributions were essential for designing and assembling the instrumentation necessary for the experimental procedures.

\section{REFERENCES}

[1] M. Akay, Time Frequency and Wavelets in Biomedical Signal Processing. Picataway, NJ: IEEE, 1998.

[2] M. G. Amin, "Time-frequency spectrum analysis and estimation for nonstationary processes," in Time-Frequency Signal Analysis: Methods and Applications, B. Boashash, Ed. Melboume, Australia: Longman Chesire, 1991, pp. 208-232.

[3] J. V. Basinajian and C. J. De Luca, Muscles Alive. Baltimore, MD: Williams \& Wilkins, 1985.

[4] H. J. Biedermann, G. L. Shanks, W. Forrest, and J. Inglis, "Power spectrum analysis of electromyographic activity: Discriminators in the differential assessment of patients with chronic low back pain," Spine, vol. 16, pp. 1179-1184, 1991.

[5] M. Bilodeau, A. B. Arsenault, D. Gravel, and D. Bourbonnais, "The influence of an increase in the level of force on the EMG power spectrum of elbow extensors," Eur. J. Appl. Physiol., vol. 61, pp. 461-466, 1990.

[6] P. Bonato, G. Gagliati, and M. Knaflitz, "Analysis of surface myoelectric signals recorded during dynamic contractions," IEEE Eng. Med. Biol. vol. 15 , pp. 102-111, 1996.
[7] H. Broman, G. Bilotto, and C. J. De Luca, "Mycelectric signal conduction velocity and spectral parameters: Influence of force and time," $J$. Appl. Physiol., vol. 58, pp. 1428-1437, 1985.

[8] H. I. Choi and W. J. Williams, "Improved time-frequency representation of multicomponent signals using exponential kernels," IEEE Trans. Acoust., Speech, Signal Processing, vol. 37, pp. 862-871, 1989.

[9] L. Cohen, Time-Frequency Analysis: Prentice-Hall, 1995

[10] T. D' Alessio, "'Objective' algorithm for maximum frequency estimation in Doppler spectral analyzers," Med Biol. Eng. Comput., vol, 23, pp. $63-68,1985$.

[11] C. J. De Luca, "Myoelectric manifestations of localized muscular fatigue in humans," CRC Crit. Rev. Biomed. Eng., vol. 11, pp. 25 1-279, 1985.

[12] —, "The use of surface electromyography in biomechanics," J. Appl Biomech., vol. 13, pp. 135-163, 1997.

[13] W. A. Gardner, "An introduction to cyclostationary signals," in Cyclostationarity in Communications and Signal Processing, W. A. Gardner Ed. Piscataway, NJ: 1EEE, 1994, pp. 1-90.

[14] S. B. Hearon and M. G. Amin, "Minimum-variance time-frequency distribution kernels," IEEE Trans. Signal Processing, vol. 43, pp. 1258-1262, May 1995.

[15] At L. Hof, "Errors in frequency parameters of EMG power spectra," IEEE Trans. Biomed. Eng., vol. 38, pp. 1077-1088, 1991.

[16] G. F. Inbar, J. Allin, and H. Kranz, "Surface EMG spectral changes with muscle length," Med. Biol. Eng. Comput., vol. 25, pp. 683-689, 1987.

[17] J. Jeong and W. J. Williams, "Kernel design for reduced interference distributions," IEEE Trans. Signal Processing, vol. 40, pp. 402-412, Feb. 1992.

[18] S. Karlsson, J. Yu, and M. Akay, "Time-frequency analysis of myoelectric signals during dynamic contractions: A comparative study," IEEE Trans. Biomed. Eng., vol. 47, pp. 228-238, Feb. 2000.

[19] M. Knaflitz, R. Merletti, and C. J. De Luca, "Inference of motor unit recruitment order in voluntary and electrically elicited contractions," $J$. Appl. Physiol, vol. 68, pp. 1657-1667, 1990.

[20] M. Knaflitz, G. Balestra, C. Angelini, and M. Cadaldini, "Muscle fatigue evaluation in the follow-up of children affected by Duchenne muscular dystrophy," Basic Appl. Myol., vol. 6, pp. 115-123, 1996.

[21] M. Knaflitz and P. Bonato. "Time-frequency methods applied to muscle fatigue assessment during dynamic contractions," J. Electromyogr. Kinesiol., vol. 9, pp. 337-350, 1999.

[22] H. Laurent and C. Doncarli, "Abrupt changes detection in the time-frequency plane," in Proc. IEEE-SP Int. Symp. Time-Frequency and TimeScale Analysis, 1996, pp. 285-288.

[23] L. Lindstrom and R. I. Magnusson, "Interpretation of myoelectric power spectra: A model and its applications," Proc. IEEE, vol. 65. pp. 653-662, 1977

[24] W. Linssen, D. Stegeman, E. Joosten, R. Binkhorst, M. Merks, H. Laak, and S. Notermans, "Fatigue in type I fiber predominance: A muscle force and surface EMG study on the relative role of type I and type II muscle fibers," Muscle Nerve, vol. 14, pp. 829-837, 1991.

[25] A. F. Mannion and P. Dolan, "Electromyograplic ınedian frequency changes during isometric contraction of the back extensors to fatigue," Spine, vol. 19, pp. 1223-1229, 1994.

[26] W. Martin, "Time-frequency analysis of random signals," in Proc. IEEE Int. Conf. Acoust., Speech, and Signal Processing, 1982, pp. 1325-1328.

[27] W. Martin and P. Flandrin, "Wigner-Ville spectral analysis of nonstationary processes," IEEE Trans. Acoust., Speech, Signal Processing, vol. ASSP-33, pp. 1461-1470, 1985.

[28] —, "Detection of changes of signal structure by using the Wigner-Ville spectrum," Signal Processing, vol, 8, pp. 215-233, 1985.

[29] R. Merletti, G. Balestra, and M. Knafliz, "Effect of FFT based algorithms on estimation of myoelectric signal spectral parameters," in Proc. 11th Conf. IEEE Eng. Med. Biol. Soc., 1989, pp. 1022-1023.

[30] R. Merletti, S. H. Roy, E. Kupa, S. Roatta, and A. Granata, "Modeling of surface myoelectric signals--Part Il: Model-based signal interpretation," IEEE Trans. Biomed. Eng., vol. 46, pp. 812-829, July 1999.

[31] A. V. Oppenheim and R. W. Schafer, Discrete-Time Signal Processing. Englewood Cliffs, NJ: Prentice-Hall, 1989.

[32] H. Piper, Electrophysillogie Muschliche Muskeln. Basel, Switzerland Verlag von Julius, 1912.

[33] A. Priez, J. Duchenne, and F. Goubel, "Duchenne muscular dystrophy quantification: A multivariate analysis of surface EMG," Med. BioL Eng. Comput., vol. 30, pp. 283-291, 1992.

[34] S. H. Roy, C. J. De Luca, and J. Schneider, "Effects of electrode location on myoelectric conduction velocity and median frequency estimates," $J$. Appl. Physiol., vol. 61, no. 4, pp. 1510-1517, 1986. 
[35] S. H. Roy, "Combined use of surface electromyography and ${ }^{31}$ P-NMR spectroscopy for the study of muscle disorders," Physical Ther., vol. 73, pp. 891-901, 1993

[36] S. H. Roy, C. J. De Luca, M. Emley, and R. Buijs, "Spectral EMG assessment of back muscles in patients with LBP undergoing rehabilitation," Spine, vol. 20, pp. 38-48, 1995.

[37] S. H. Roy, P. Bonato, and M. Knaflitz, "EMG assessment of back muscle function during cyclical lifting," J. Electmomyogr. Kinesiol., vol. 8, pp. 233-245, 1998

[38] A. M. Sayeed and D. L. Jones, "Optimal kemels for nonstationary spectral estimation," IEEE Trans. Signal Processing, vol. 43, pp. 478.491, Feb. 1995.

[39] F. Sulen and C. J. De Luca, "Frequency parameters of the myoelectric signal as a measure of muscle conduction velocity," IEEE Trans. Biomed. Eng., vol. BME-28, pp. 512-523, 1981.

[40] L. B. White and B. Boashash, "On estimating the instantaneous frequency of a Gaussian random signal by use of the Wigner-Ville distribution," IEEE Trans. Acoust., Speech, Signal Processing, vol. 36, pp. 417-420, Mar. 1988

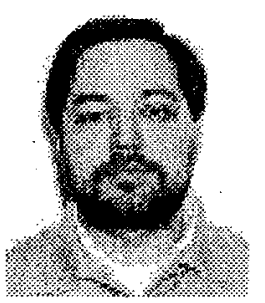

Paolo Bonato received the Italian Laurea degree in electrical engineering from Politecnico di Torino, Torino, ltaly, in 1989. From 1990 to 1991 he was a research fellow with the Biophysics Research Group of IRST, Trento, Italy where he was active in the field of signal processing applied to the cardiovascular system. From 1992 to 1994 , he was a Ph.D. degree candidate at the Dipartimento INFOCOM of the Università di Roma "La Sapienza," Roma, ltaly. His work addressed the analysis of surface myoelectric signals recorded during dynamic contractions. After he received the $\mathrm{Ph} . \mathrm{D}$. degree in biomedical engineering, he was granted a post-doctoral fellowship at the Dipartimento di Elettronica of Politecnico di Torino, Torino, Italy.

Since 1996, he has been Research Assistant Professor at the NeuroMuscular Research Center of Boston University, Boston, MA

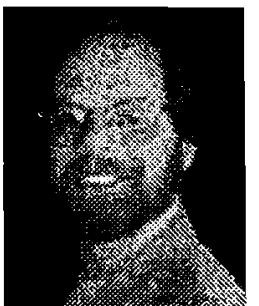

Serge H. Roy received the B.S. degree in physical therapy from New York University (N.Y.U.), New York, in 1975, the M.S. degree in physical therapy from Boston University, Boston, MA, in 1981, and the Sc.D. degree from Boston University in applied anatomy and kinesiology in 1992.

He is currently Research Associate Professor at the NeuroMuscular Research Center (NMRC) and the Sargent College of Health and Rehabilitation Sciences at Boston University. At the NMRC, he supervises the Muscle Fatigue Laboratory (1984) and the Electrophysiology Laboratory (1989). His work is directed at developing surface electromyographic (EMG) systems for the assessment of muscle impairments in patients with musculoskeletal disorders. He is also a Research Associate for the Department of Veterans Affairs since 1988. His principal research interests include signal processing applied to the biomedical field and rehabilitation engineering, analysis of nonstationary signals by time-frequency analysis, electromyography applied to kinesiology, and biomechanics of movement.

Dr. Roy is the recipient of the Founder's Day Award for Scholarship and the Elizabeth C. Adams Award (N.Y.U.; 1974 and 1975), and two Group Achievement Awards from the National Aeronautics and Space Administration for Spacelab Life-Science Missions (1992 and 1994). He was recently awarded a Fellowship to the American Institute for Medical and Biological Engineering (AIMBE) and is President of the International Society of Electrophysiology and Kinesiology (ISEK). He is a Menber of IEEE EMBS and of IEEE SP societies.

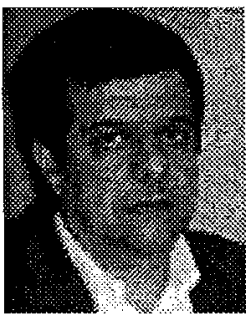

Marco Knaflitz received the Italian Laurea in electrical engineering and the Ph.D. degree in electronics from Politecnico di Torino, Torino, Italy.

From 1989 to 1990, he was a Research Assistant Professor at the NeuroMuscular Research Center of the Boston University, Boston, MA. Since 1990, he is with the Department of Electronics of Politecnico di Torino, where he currently is Associate Professor of Biomedical Engineering. His research interests include various aspects of biomedical signal processing as well as the design of biomedical instrumentation. with specific reference to the neuromuscular system. He has an extensive experience on the safety aspects related to the design, management, and use of biomedical instrumentation.

Dr. Knaflitz is a Member of IEEE EMBS, ISEK, AIIMB (affiliated with IFMBE), and SIAMOC.

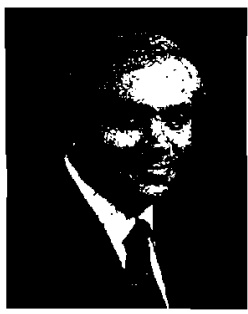

Carlo J. De Luca received the B.A.Sc. degree in electrical engineering from the University of British Columbia, Vancouver, B.C., Canada; the M.Sc degree with specialization in biomedical engineering from the University of New Brunswick, Fredericton, N.B., Canada; and the Ph.D. degree from Queen's University, Kingston, ON, Canada.

$\mathrm{He}$ is Director of the NeuroMuscular Research Center at Boston University, Boston, MA. After beginning his academic career at Queen's University, he was on the faculty of Massachusetts Institute of Technology and Harvard Medical School simultaneously from 1974 to 1984. He then moved to Boston University where he was appointed as tenured Professor in Biomedical Engineering and was made Director of the NeuroMuscular Research Center, which he founded. In 1985, he was also appointed as Research Professor of Neurology. From 1986 to 1989 he was the Dean of the College of Engineering. His tesearch has been centered on issues dealing with rehabilitation of the physically disabled. His early interest focused on control of externally powered prosthetics. Currently, he is working on understanding how the brain and spinal cord control the individual fibers in a muscle, and groups of muscles, in healthy as well as dysfunctional individuals. His research also includes methodologies for objectively measuring muscle fatigue during voluntary efforts and means for monitoring functional activities. He is aslo developing new technologies for detecting and interpreting EMG signals. 
